\title{
Correction and Extension of WordNet 1.7
}

\author{
Philippe Martin \\ Distributed System Technology Centre \\ Griffith University, PMB 50 Gold Coast MC, QLD 9726 Australia \\ philippe.martin@gu.edu.au
}

\begin{abstract}
This article presents the transformation of the noun-related part of WordNet into a genuine "lexical ontology" to support knowledge representation, sharing and retrieval within a knowledge base or on the Web, i.e. to support "knowledge creation and communication". The corrections and extensions are documented at http://www.webkb.org/doc/wn/ and the ontology is downloadable in various formats. Web users can also search and extend the ontology via the WebKB-2 knowledge server.
\end{abstract}

\section{Introduction}

WordNet [1] is a lexical database that connects English words to "synonym sets" (each "synset" represents one of the meanings of the words in the set) and organizes the synsets by semantic links, e.g. specialization and partOf links. WordNet is increasingly interpreted and exploited as a lexical ontology (i.e. a set of categories connected by links having a formal semantics) despite its shortcomings for this purpose.

A natural language ontology derived from WordNet and other sources could support or enhance various kinds of applications, e.g. query expansion and answering [5], machine translation [8], and knowledge representation, sharing or brokering [3] [4]. In [11], I argued that the Semantic Web (as I understand it) cannot be achieved without at least one natural language ontology that can be extended by people and permit to give categories from different ontologies some shared meaning. [11] also details how the knowledge server WebKB-2 exploits WordNet 1.7 and its extensions for guiding and checking knowledge representation, and for permitting Web users to share or retrieve knowledge, and further extend or correct the shared ontology if necessary. (Protocols and naming conventions prevent lexical and semantic conflicts).

This article introduces extensions and corrections of the noun-related part of WordNet 1.7 to transform it into a lexical ontology usable for knowledgebased applications, and especially the manual representation of natural language sentences. (Much more would be needed to support natural language parsing) ${ }^{1}$.

\footnotetext{
${ }^{1}$ Only the noun-related part of WordNet 1.7 is used because the use of categories representing the meanings of verbs, adverbs or adjectives has several drawbacks: (i) using such categories with quantifiers has no real meaning (e.g. "any transformation" or "3 transformation" has a meaning but "any transform" and "3 transform" has not), (ii) organizing these categories by generalization links is difficult or impossible, (iii) these categories are (non-defined) shortcuts for more explicit constructions using categories for nouns. More details and rationales can be found in [11].
} 
No claim is made that this ontology is sufficient to support the inter-operation of fully automatic software agents, e.g. for e-commerce or database integration purposes. [6] shows that such inter-operations have strong requirements and, in the general case, are not likely to be fully supported by ontologies anytime soon.

This article first explains why short and intuitive identifiers were generated for each WordNet category, and illustrates the lexical corrections. Second, it explains how types (1st-order categories) were distinguished from individuals (0th-order categories), and hence how WordNet specialization links were differentiated into subtype and instance links. Third, it introduces the top-level ontology of concept and relation types into which the top-level categories of WordNet were inserted to support the construction of normalized (i.e. better retrievable) knowledge statements and certain semantic checks on the ontology and the statements. Fourth, it illustrates the kinds of problems that led to the removal or modification of links in WordNet. Fifth, it details the kinds of additions (links, schemas, annotations) made to some WordNet categories.

\section{Category Identifier Generation and Lexical Corrections}

A category may have many names (the elements of the "synset" in WordNet) that may be shared by other categories, but should have at least one "identifier" to refer to it uniquely. In WebKB-2, a category identifier is allowed to be a URL or an e-mail address, but for readability reasons, is most often composed of a short identifier for the user (or source) that created the category, and a key name distinguishing the category from other ones created by the same user. For example, wn\#car refers to a WordNet category for the noun "car", while pm\#car may represent a different notion for the user pm. WebKB-2 allows the prefix "wn" to be dropped, and the category creator may specify other names by appending them to the identifier; thus, \#car__auto__automobile refers to the same category as wn\#car. (Such categories may also be referred and accessed from outside WebKB-2 via a URL. The reader is encouraged to access http://www.webkb.org and browse from the categories referred to in this article).

WordNet has at least two internal identifiers for each category, e.g. the category for "Friday" has for identifiers 12558316 and friday\%1:28:00:: . While some applications re-use them, others (such as [2]) generated their own identifiers by concatenating names or using suffixes, e.g. Inessential\$Nonessential and Cell_1. However, for knowledge representation, exchange and sharing purposes, category identifiers should be concise and clear to permit readable text-based knowledge statements (graphical interfaces should not be required and are not necessarily the best device to enter, display, debug or maintain a large amount of knowledge [11]; category identifiers should also be usable within controlled languages). Hence, for these purposes, each category should have at least one identifier composed of a common and unambiguous word or expression for its meaning, and as little else as possible. This means that one of the category names should be used as key name, if possible with no suffix. This was possible for $92 \%$ of WordNet categories related to nouns:only 5944 WordNet categories (out of 
$74,488)$ have been given a key name with a suffix. The list of these categories and the used identifier generation algorithm is accessible from [15].

So is the list of my 353 lexical corrections: 28 modifications of category annotations, 248 category names added, and 77 manual re-orderings of category names. Here is an example showing how the corrections were documented:

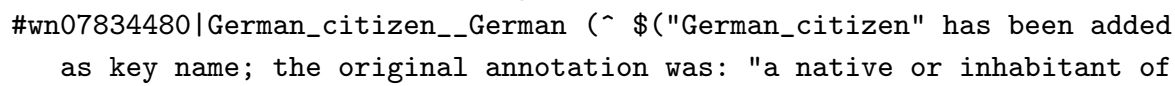

This format is used by WebKB-2 for saving the KB in a backup file. 07834480 is the WordNet identifier, German_citizen the added key name (since "German" also refers to a language), German the original name, $\left({ }^{\wedge} \ldots^{\wedge}\right)$ the category annotation, and $\$(.) \$.$$ a sub-annotation which WebKB-2 does not show to end-users.$

To conclude, this work provides Web users a shared formal vocabulary to mark up their documents or the meanings of words in their documents, or to use in their knowledge statements. If a word meaning is missing, a user may easily add it to the ontology via WebKB-2, thus permitting other people to retrieve and re-use his/her categories or statements.

\section{Explicitation of Individuals}

Distinguishing 1st-order types from their instances ("individuals"), is important for knowledge representation, inferencing and checking. Individuals cannot have specializations, i.e. subtypes or instances. Certain individuals, often called continuants or endurants [2], can change in time without being viewed as different individuals (i.e. without loosing their identity), e.g. individuals for persons or cities. Specializing such individuals according to time might be tempting, e.g. pm\#ParisIn1995, but better avoided: statements (facts or definitions) about individuals should represent dates and durations in an explicit way using contexts.

Distinguishing types from individuals is not always obvious. For example, [2] asserts that the WordNet category \#karate should be an individual, but there are various kinds of karate, and furthermore, since \#karate is a subtype of \#activity [2], each individual practice of karate may be considered as an instance. Anything that may be specialized, or has various occurrences, or comes in different variants or versions should be represented as a type rather than an individual; otherwise, knowledge representation possibilities and accuracy are reduced. For example, any doctrine, book, language, alphabetic character, code, diploma, sport or recurring situation should rather be represented as a type. The first character of the alphabet has many variants (e.g. its uppercase and lowercase variants) and billions of instances (occurrences) in books. An alternative view would be to consider that in certain cases a variant is not a subtype and an occurrence is not an instance, and then use different links or relations to represent this information. However, in this alternative model, information would be more complex to describe, and inferencing more complex to implement.

I chose the simplest model. However, since people often wish to use certain types without quantifiers, as if they were individuals (e.g. in English, the nouns 
"Monday" and "Polish" are rarely used with an article, i.e. a quantifier), WebKB2 allows it in Frame-CG (FCG) and Formalized-English [10] (both extend and simplify the Conceptual Graph Linear Form (CGLF)) on the condition that the category has no subtype, no instance and is not a subtype of pm\#physical_entity nor \#time_period.

[15] lists the 6211 individuals that I manually isolated: typically, time periods, persons, organizations, places and battles. To do so, I first translated all WordNet specialization links as subtype links. Then, since WordNet categories are grouped by theme within the WordNet database files, I operated a careful but relatively quick "search and replace" of subtype links into instance links in the zones where individuals could appear. I double checked this work on categories having a name with a capitalized first letter. Here is an example in the FO notation (which is also derived from CGLF; ' ' represents the instanceOf link and 'P' the WordNet partOf link): \#Neolithic_Age - \#time_period, P \#Stone_Age;

To sum up, formal information was added to WordNet categories (consistent with the original meanings of these categories) while adopting an approach that maximise re-use possibilities. For knowledge sharing and inferencing purposes, I also argue against the use of instance links between types (i.e. against the introduction of second-order types and second-order statements) when subtype links can be used instead. Indeed, subtype links are easier to use for structuring categories, and then to exploit. The logical interpretation of statements using types of different orders may also be difficult and they are not commonly exploited by inference engines. Over-uses of the instance link are frequent. For example, the TAP KB [14] categorizes certain types of magazines or books as instances of a second-order type tap\#product_type which has no other supertype than rdfs\#class. Even if it had, the use of a first-order type such as \#product permits much more comparison with (or connection or inheritance of constraints from) other types, hence more retrieval and checking possibilities.

\section{Top-level Ontology}

WordNet has not been built for knowledge representation purposes, nor apparently according to basic taxonomy building principles and with consistency checking tools. As noted in [2], types and individuals are not distinguished; the annotation of a category is not to be relied on as it may be contradicted by specializations of this category; direct specializations often have heterogeneous levels of generality; role types (e.g. \#student) are not distinguished from natural types (e.g. \#person) and may generalize them. I also found that (i) specialization links are sometimes used where "location" or "similar" links should be used, (ii) the "part" and "member" links between types are not used in a consistent way (most seem to mean that all instances of the source type have for part/member at least one instance of the destination type, but this is not always the case), (iii) some of these transitive links are redundant, and (iv) exclusion links are sometimes broken, i.e. some exclusive categories have common specializations. Table 1 shows the uppermost WordNet categories for nouns and some of their direct subtypes. The lack of structure is clear. 
Table 1. WordNet 1.7 top categories for nouns (brackets enclose exclusive subtypes)

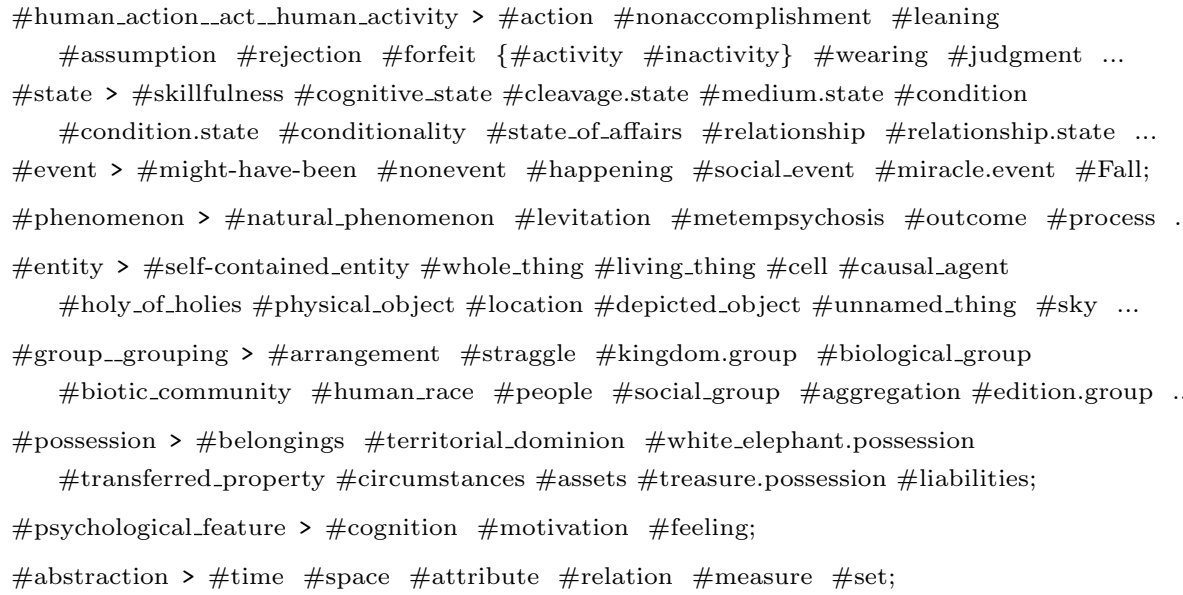

This work seems the first to have isolated individuals, generated intuitive category identifiers, corrected and documented a large number of problems, and permitted Web users to further extend and correct this ontology. No attempt to bring more structure to the whole of WordNet was made, as this would probably take many years. However, like others, this work inserts the top-level categories of WordNet into a better structured top-level ontology ("top-level" simply means "general" without arbitrary notions of "primitiveness" or "depth"; time is the main limiting factor since, outside particular applications, the more specialized the categories, the less their (re)structuration is likely to be useful). In 1994, Sensus [8] was created by manually merging the WordNet top-level into Ontos and the Generalized Upper Model, and then semi-automatically merging WordNet with the Longmann Dictionary of Contemporary English. Sensus was created for machine translation purposes. At the same period, for knowledge acquisition and representation purposes, I extended Sowa's first top-level ontology [12] and used it for structuring WordNet 1.5 top-level [9]. In 2001, for the Semantic Web and other knowledge sharing purposes, the OntoClean ontology and methodology was used to re-structure WordNet 1.6 top-level [2]. In October 2002, I integrated the last version of the OntoClean ontology, DOLCE (D17) [16] into WebKB-2 ontology but found most of the 40 DOLCE top categories too specific to specialize them with WordNet categories. The next section presents two examples.

\subsection{Minimizing Re-categorization}

Example 1. OntoClean/DOLCE distinguishes "qualities" (like size, color, redness, smell and duration) from "quales" (quality regions/spaces, i.e. categories of values for qualities, e.g. \#red, \#past_times and \#Greenwich_Mean_Time [2]). They specialize the exclusive categories dolce\#quality and dolce\#region_-quale. 
However, in WordNet, such categories (about 8900) are inter-related by specialization links, e.g. \#red__redness specializes \#chromatic_color and \#color, while \#past_times specializes \#time. Hence, specializing the types dolce\#quality and dolce\#region by WordNet categories, as suggested in [2], is problematic: (i) this classification has to be done for most of the 8900 categories, not just for their most general categories; (ii) a great number of WordNet specialization links have to be broken, hence this structure is lost and the meaning of a great number of WordNet categories is modified; (iii) it is often difficult to decide whether a WordNet category should be interpreted as a quality or as a quale; as opposed to [2], I consider \#Greenwich_Mean_Time, \#work_time and \#red as quality types (the authors of [2] argue for the representation of red and other adjectives for colors as quales, but \#red (i.e. \#red__redness) represents the meaning of the nouns "red" and "redness"). In my integration of WordNet, I added or refined but not removed or modified links - except for 306 (out of 74,488 ) in order to fix inconsistencies. From an Ontoclean perspective, this is possible by interpreting most of the above cited 8900 categories as qualities. However, I have not explicitely categorized their upper types as specializations of dolce\#quality in order to permit WebKB2 users to classify certain WordNet categories as subtypes of dolce\#region when this does not introduce inconsistencies. I have generalized these upper types, plus dolce\#quality and dolce\#region, by the type pm\#attribute_or_measure (this name is due to the fact that things I call "measures" may specialize the things that are often called "attributes").

Here is a statement in FCG showing how knowledge representation can be done in an intuitive and normalizing way with the interpretation of WordNet attributes or measures as qualities: [a \#car, \#color: a \#red, \#weight: 900 \#kg]. In Formalized-English [10]: there exists a \#car that has for \#color some \#red and for \#weight 900 \#kg. Both \#red and \#kg are quantified (KIF definitions for the FCG numerical quantifiers are given in [10]). As in Ontoseek [3] (a WordNetbased knowledge retrieval system built by the team that designed OntoClean), the types \#color and \#weight are used as if they were relation types. WebKB-2 checks that these types specialize pm\#thing_that_can_be_seen_as_a_relation and respectively generalize \#red and \#kg. No quale is explicitely referred to in this statement. If \#red and \#kg were categorized as quales, more complex statements would have to be written, e.g.: [a \#car, \#color: (a \#color, pm\#measure: a \#red), \#weight: (a \#weight, pm\#measure: 900 \#kg)]. Checking this graph would also be more complex and would require additional information on categories acceptable as measures for colors and weight.

Example 2. In [2], \#substance is subtype of dolce\#amount_of_matter which is exclusive with dolce\#physical_object However, \#substance has many subtypes which are also subtypes of dolce\#physical_object. An example is \#olive.relish which specializes \#fruit (\#physical_object) and \#relish (\#condiment, \#substance). Another example is \#glass_wool, subtype of \#artifact (\#physical_object) and \#insulator (\#substance). Since these WordNet links do not appear as clear mistakes, it seems that in [2], \#substance has been over-interpreted (or adapted) to fit the meaning of dolce\#amount_of_matter. Instead, I categorized \#substance 
(along with other types such as \#physical_part and \#building_block) as subtype of pm\#physical_part_or_substance which, like dolce\#physical_object and dolce\#amount_of_matter, is a direct subtype of pm\#physical_entity. Since this last type covers both substances and physical objects, it may be seen as an adequate candidate for classifying a "statue of clay". It may also be used for signatures of relations, e.g. relations representing physical attributes such as color or mass (although as hinted in Example 1, this is discouraged in WebKB).

\subsection{Summary of the Approach and its Results}

The distinctions made by DOLCE and other top-level ontologies are important and their integration may be used by knowledge servers to guide the users to represent knowledge in more precise and re-usable ways. The precision of DOLCE categories and their associated constraints are also intended to ease the automatic matching of categories from (Semantic Web) ontologies independently developed but re-using the DOLCE ontology. Although this precision makes the current set of DOLCE categories difficult to use for structuring WordNet top-level (and other distinctions are also required), it is valuable (including the distinction between qualities and quales, although in my approach I am more interested in the more general distinction between concept types that can be used for relations and those that can be used as destinations of those relations; Section 6 will show how I have prefered to make the distinction).

Table 2 presents a summary of top-level types ${ }^{2}$. Many types from WordNet, DOLCE and Sowa are shown. The catch-all WordNet categories \#entity and \#abstraction do not appear but their direct subtypes have been categorized in various places. Most of the upper types, e.g. pm\#spatial_entity or pm\#description, are common and relatively intuitive categories that are required for the signatures of the relation types (Table 3 ). These concept types have been given constraints (mainly exclusion links) and prototypes (e.g. typical relations) that are inherited by their numerous WordNet specializations.

The relation types proposed by WebKB-2 are mainly for primitive binary relations and intended to support an explicit and normalized way of representing natural language sentences (in [11], I give rationales against the use of nonbinary relations and complex relations, e.g. relations representing processes). I also integrated argumentation relations and the relations of DAML, RDF, RDFS, Dublin Core and the core of KIF. Table 3 shows the overall organization, although it also deepens in the case relations. The grouping by source category proved to be the cleanest and most intuitive structure, and WebKB-2 exploits it when generating menus to guide knowledge representation.

The "Suggested Upper Merged Ontology" (SUMO) [17] has similarities with WebKB-2 ontology in the sense that it has mappings with categories of WordNet 1.6, and includes some spatial and case relations, and various top concept

\footnotetext{
${ }^{2}$ To be compatible with most other top-level ontologies, the uppermost type has for subtypes all other categories, including relation types and second-order types. Hence, Table 2 does not just present concept types. Although this is not certain, it does not seem that the top types of Sowa's ontology and DOLCE are concept types only.
} 
Table 2. Some of the 160 top-level types in WebKB-2

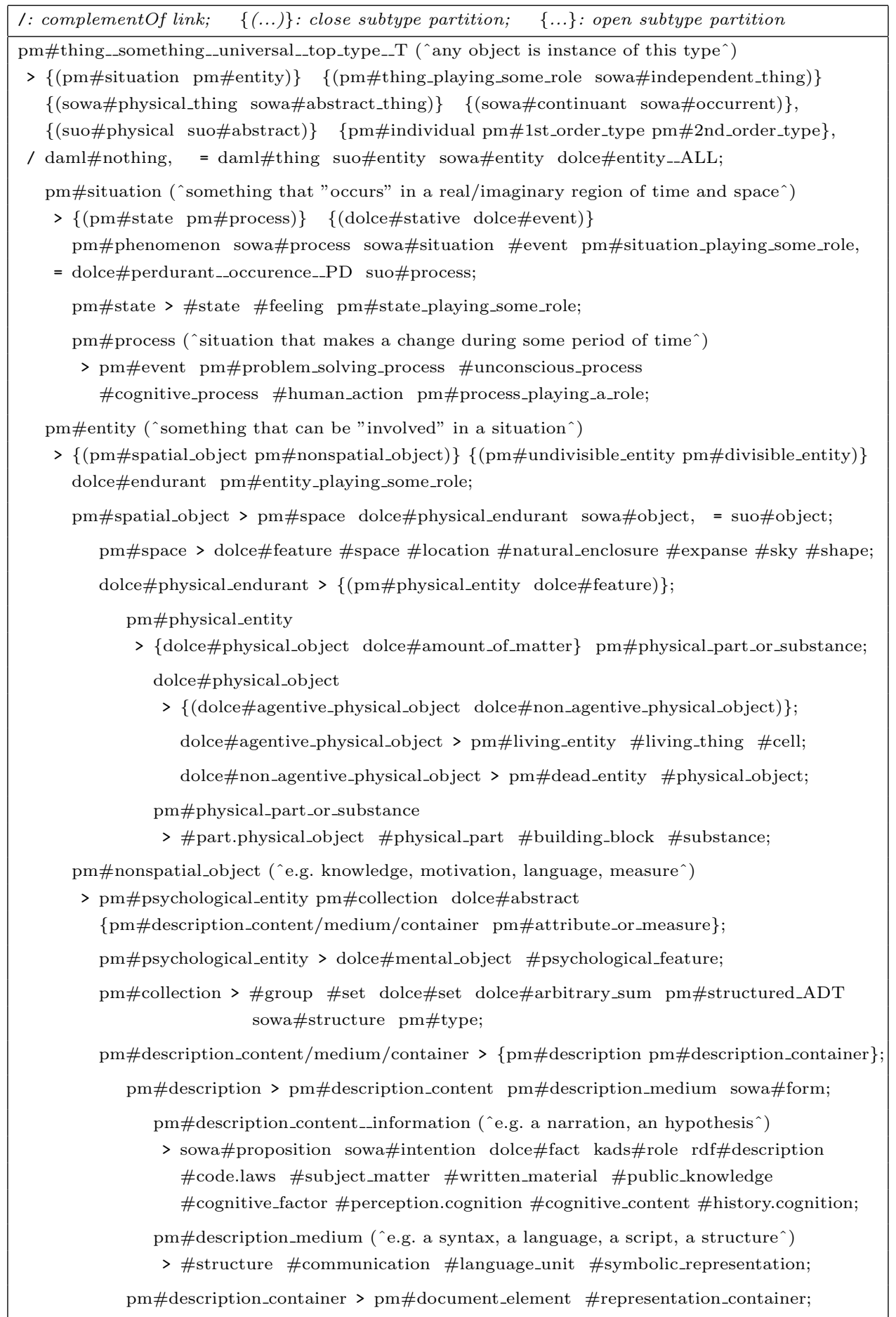


Table 3. Some of the 150 primitive relation types in WebKB-2

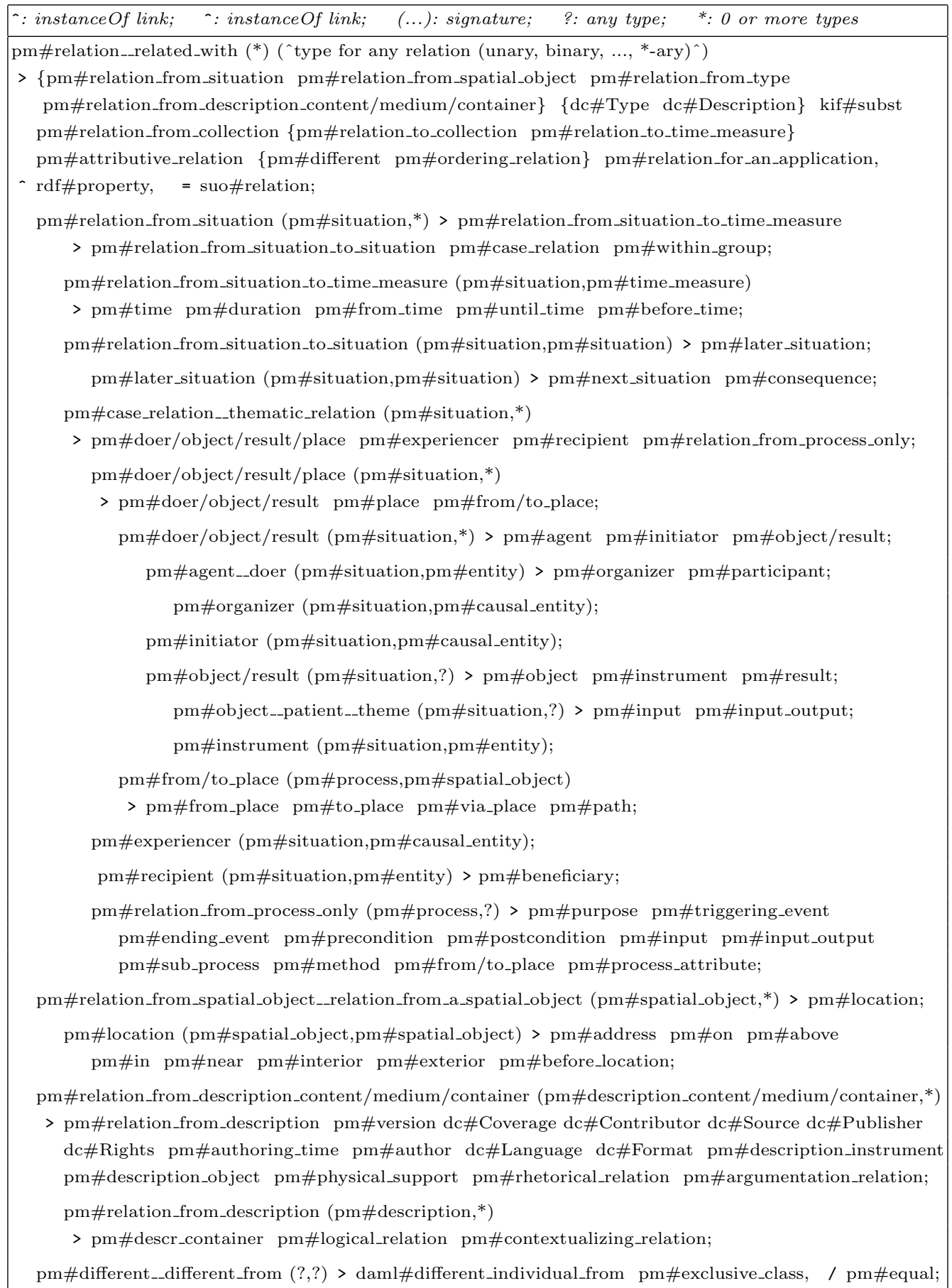


types from various top-level ontologies, e.g. Sowa's last top-level ontology. Its integration into WebKB-2 ontology has begun. Some elements of the CYC toplevel [18] may also be added in the future (only some elements since on the one hand, there is already some overlap, and on the other hand different approaches have been adopted in CYC, e.g. it includes many non-binary relations and relations representing processes).

To conclude, WebKB-2 and its ontology may help people avoid the difficult task of finding, integrating and extending adequate ontologies, especially toplevel ontologies (a task that some Semantic Web researchers, seem to think the knowledge providers to the future Semantic Web are able to do, have the time to do and will have to do! [7]). Instead, the WebKB-2 user is simply supposed to find adequate categories by typing words and browsing from the proposed categories for these words, and then fill cascading menus adapted to the categories s/he selected or entered. Knowledge precision and normalization is encouraged by the various proposed distinctions, the adopted approach (e.g. the proposed basic binary relations) and the proposed notations (e.g. their extended quantifiers).

\section{$5 \quad$ Semantic Corrections}

Up to March 2003, 117 links have been removed, and the types or destinations of 198 links have been modified. Of these 315 links, 41 were redundant and about 240 inconsistent with other links. Most of the inconsistencies were automatically detected thanks to exclusion links in WebKB-2 top-level ontology. For example, some categories in WordNet were classified as both human action and causal agent, instrument or result of action (e.g. \#relaxant and \#interpretation) or of communication medium/content (e.g. \#epilog and \#thanksgiving), or as both communication medium/content and physical entity (e.g. \#book_jacket) or attribute (e.g. \#academic_degree). Some WordNet specialization links were also used instead of "member" links, (e.g. between types for species and genus of species). Similarly, WordNet does not have "location", "similarTo" and "identity" links, and uses subtype links instead of location links (e.g. many city/regions where battles have occured were classified both as city/regions and battles), similarTo links (e.g. for a Greek god and its Roman counterpart) and identity links (WordNet introduces a few categories to represent obsolete names).

Redundancy was detected by exploiting the transitivity of specialization, part and member links. Only the combination of exclusion and specialization links was exploited to detect inconsistencies or redundancies. More could be done. For example, the fact that "if t2 specializes t1, and t1 is member of t0, then t2 is member of t0" should be exploited to detect more redundant links, e.g. in WordNet both \#dog and its subtype \#hound_dog are member of \#pack.animal_group. Negative constraints such as "if t2 specializes t1, then t2 cannot be linked by any other kind of link to t1" have not been exploited either but it does not seem that WordNet 1.7 has many problems of this kind.

The corrections I made are documented [15]. Table 4 shows some examples in the format used for saving the KB of WebKB-2 in a backup text file. 
Table 4. Examples of corrections

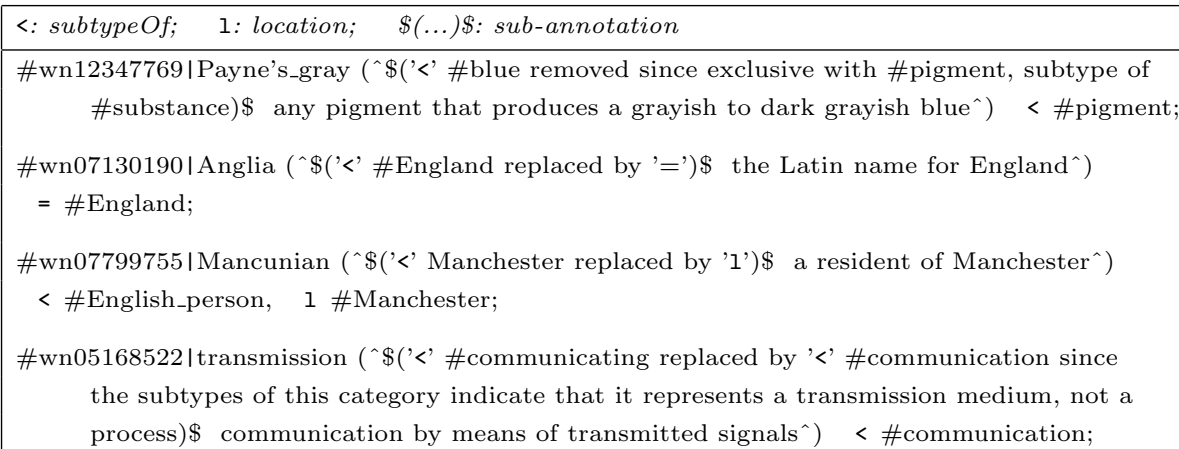

\section{Additions}

Up to March 1993 (and apart from the connections of WordNet upper categories to my top-level concept types), I have added 161 links, 17 during the integration of WordNet to WebKB-2 and 144 later when using the ontology for representing knowledge (thus, this excludes the 3000 specializations of WordNet categories that I created for specific applications/domains, e.g. information technology). About 65 of these links connect WordNet categories, while 90 connect a WordNet category to a new specialization. Table 5 shows some examples.

Table 5. Examples of link additions

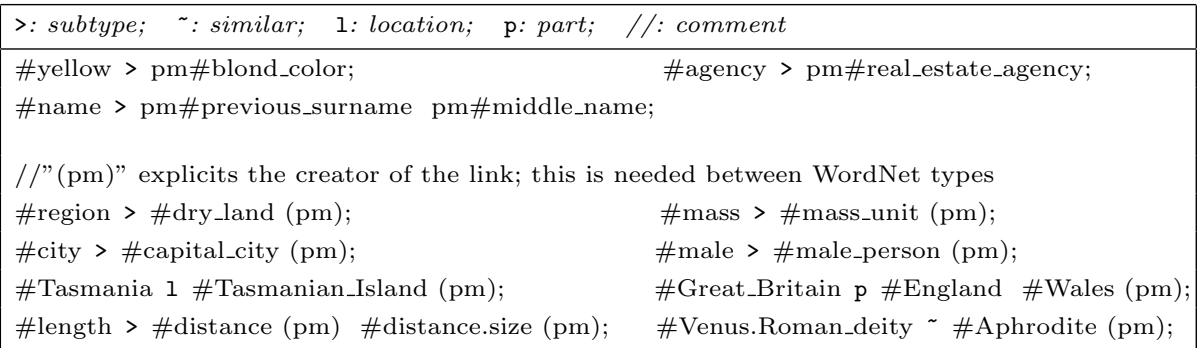

I also added sub-annotations to guide or check knowledge entering. For example, since I do not want to distinguish between qualities and quales using subtype links, the subtypes of pm\#attribute_or_measure that represent values need to be distinguished in another way to prevent them being used within relations (or proposed in WebKB-2 generated menus for relations). Hence, I checked all the subtypes of pm\#thing_that_can_be_seen_as_a_relation) and added the string $\$$ (value) $\$$ in the annotations of about 1300 of them. (It should be noted that individuals are representing values and hence such sub-annotations are not required for them). The string $\$$ (artificial) $\$$ was also added in the annotations of 
WordNet categories that were found unfit for knowledge representation purposes, generally because they had a lexical rather than semantic character. Table 6 gives some examples.

Table 6. Examples of value/artificial categories

\begin{tabular}{|c|c|}
\hline $\begin{array}{l}\text { \#dark_red }(` \$(\text { value }) \$ \text { a red that reflects } \\
\left.\text { little light }{ }^{\wedge}\right)\end{array}$ & $\begin{array}{l}\text { \#then }(\wedge \$(\text { artificial }) \$ \text { that time; } \\
\text { "we will arrive before then"^) }\end{array}$ \\
\hline $\begin{array}{l}\text { \# } \text { gram }_{--} \text {gramme_-gm }- \text { g }(\wedge(\text { value }) \$ \text { metric unit } \\
\left.\text { of weight equal to one thousandth of a } \mathrm{kg}^{\wedge}\right)\end{array}$ & $\begin{array}{l}\text { \#thing.action ( } \$ \text { (artificial) } \$ \text { action } \\
\text { "how do you do such a thing?"^) }\end{array}$ \\
\hline $\begin{array}{c}\text { \#west_by_south_-WbS }(\wedge \$(\text { value }) \$ \text { the compass } \\
\left.\text { point that is one point south of due west }{ }^{\wedge}\right)\end{array}$ & $\begin{array}{l}\text { \#thing.happening ( } \$(\text { artificial }) \$ \text { an } \\
\text { event; "something happened ..."^) }\end{array}$ \\
\hline $\begin{array}{l}\text { \#Monday__Mon ( }(`(\text { value }) \$ \text { the second day of } \\
\text { the week; the first working day^) }\end{array}$ & $\begin{array}{l}\text { \#tonight }(` \$(\text { artificial }) \$ \text { the present } \\
\text { or immediately coming night^) }\end{array}$ \\
\hline \#andante $(` \$($ value $) \$$ moderately slow tempo^) & \\
\hline \#mealtime (^\$(value) $\$$ time $\mathrm{f}$ & \\
\hline
\end{tabular}

Finally, I entered statements representing the most common relations that are or may be associated to certain categories. I call them schemas. Table 7 shows an example in FCG. WebKB-2 exploits such schemas to generate menus that help users to search or represent knowledge. Figure 1 shows an example based on the schema in Table 7 and where only this schema is exploited because of its sub-annotation $\$$ (no inheritance) \$. The sub-annotation \$(explore)\$ in a relation annotation directs WebKB-2 to present the subtypes of the type used for the relation, in a select menu (except for the subtypes marked as "value" or "artificial"). The '+' symbols in the menus permit the user to access a sub-menu to detail relations from/to any destination object $\mathrm{s} /$ he has entered; in other words, menus can be cascaded to guide the entering of a query or statement.

Table 7. Example of schema

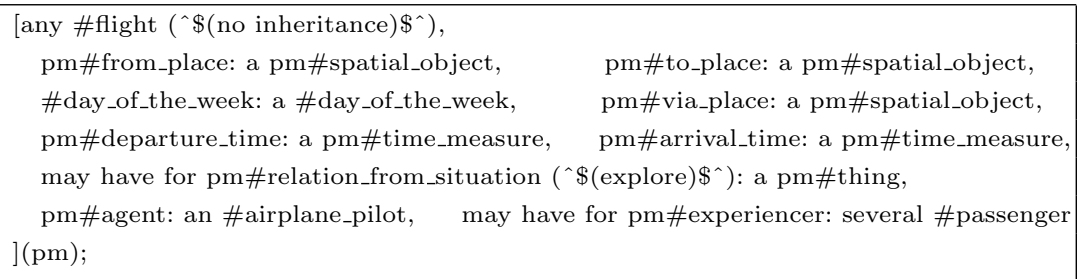




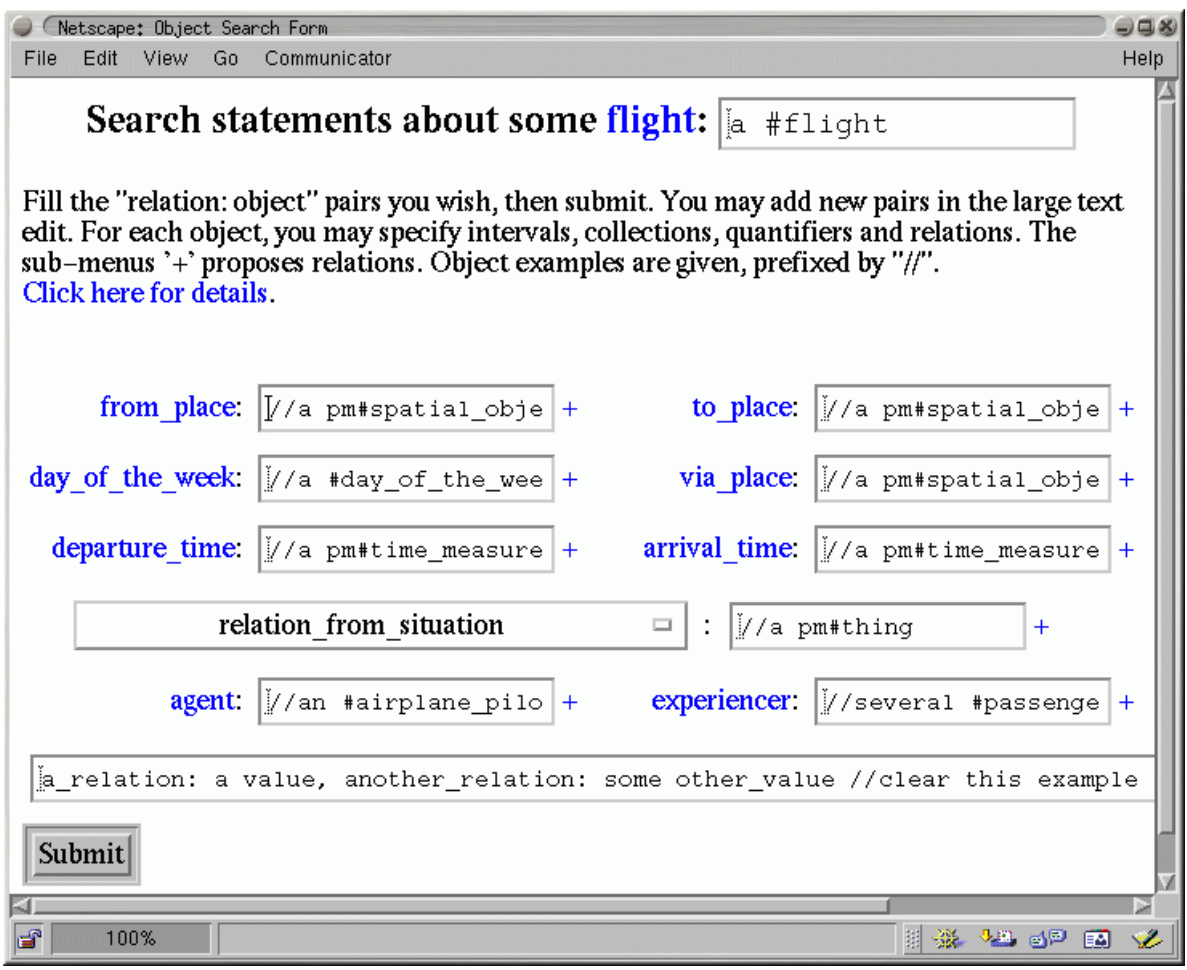

Fig. 1. A generated menu to help searching flights in the knowledge base.

\section{Conclusion}

The noun-related part of WordNet has been transformed into a genuine "lexical ontology" usable as a component in various knowledge-based applications: metadata registries, Yellow-pages like catalogs, query expansion, Semantic Web, etc. The focus was to guide and ease the representation, retrieval and sharing of general knowledge. This involved the generation of readable and unambiguous identifiers, the extraction of individuals, the merge with various top-level ontologies, and the correction of lexical and semantic problems. The result ontology is downloadable, browsable and extendible by anyone at http://www.webkb.org/.

Although I structured the top-level of WordNet and added a few links in other parts, the direct specializations of nearly all WordNet categories remain quite heterogeneous, with few exclusion links, and without distinction between role types and natural types. This lack of structure may be a problem for certain applications but fixing it may be as difficult as creating a better WordNet from scratch. Another problem is that distinctions in WordNet seem to have often been made not simply on semantic grounds but also on lexical grounds, thus leading to a multiplicity of "artificial" categories or categories that should be connected but are not. A few categories have been marked as "artificial" but 
many more would need to be similarly marked, or connected by specialization links, to improve knowledge normalization and retrieval.

The next step is to integrate other ontologies from the IEEE Standard Upper Ontology library [19], in particular the Suggested Upper Merged Ontology (SUMO), and the DAML Ontology Library [20], in particular the CIA World Factbook. In the mapping that has been done between the SUMO and WordNet, one SUMO categories is often linked to several WordNet categories. That will give cues to find and mark many WordNet categories as "artificial".

\section{References}

1. G. Miller. Wordnet: a Lexical Database for English. Communications of the ACM, 11:39-41, 1995. http://www.cogsci.princeton.edu/ ${ }^{\sim}$ wn/

2. A. Gangemi, N. Guarino and A. Oltramari. Restructuring Wordnet's Top-Level. AI Magazine, 40(5):235-244, fall 2002.

3. N. Guarino and G. Vetere. Ontoseek: Content-based Access to the Web. IEEE Intelligent Systems, 14(3):70-80, October 1999.

4. A. Puder and K. Romer. Generic Trading Service in Telecommunication Platforms. In Proceedings of ICCS'97. LNAI 1257:551-565, August 1997.

5. C. Kwok, O. Etzioni and D. Weld. Scaling Question Answering to the Web. ACM Transactions on Information Systems, 19(3), July 2001.

6. R. Colomb. Impact of Semantic Heterogeneity on Federating Databases. The Computer Journal, 40(5):235-244, 1997.

7. J. Hendler. Agents and the Semantic Web. IEEE Intelligent Systems, 16(2), 2001.

8. K. Knight and S. Luk. Building a Large-Scale Knowledge Base for Machine Translation. In Proceedings of AAAI'94, 773-778, Seattle, USA, July 1994.

9. P. Martin. Using the Wordnet Concept Catalog and a Relation Hierarchy for Knowledge Acquisition. In Proceedings of Peirce'95, Santa Cruz, CA, August 1995. http://www.webkb.org/doc/papers/peirce95/

10. P. Martin. Knowledge Representation in CGLF, CGIF, KIF, Frame-CG and Formalized-English. In Proceedings of ICCS'02, LNAI 2393:77-91. http://www.webkb.org/doc/papers/iccs02/

11. P. Martin. Knowledge Representation, Sharing and Retrieval on the Web. Web Intelligence (Eds.: N. Zhong, J. Liu, Y. Yao). Springer-Verlag, January 2003. http://www.webkb.org/doc/papers/wi02/

12. J. Sowa. Conceptual Structures: Information Processing in Mind and Machine. Addison-Wesley, Reading, MA, 1984.

13. J. Sowa. Knowledge Representation: Logical, Philosophical, and Computational Foundations. Brooks Cole Publishing Co., Pacific Grove, CA, 2000. See also http://users.bestweb.net/ sowa/ontology/

14. R. Guha and R. McCool. TAP: a system for integrating web services into a global knowledge base. 2002. http://tap.stanford.edu/

15. The WordNet 1.7 integration documentation. http://www.webkb.org/doc/wn/

16. The DOLCE Ontology. http://wonderweb.semanticweb.org/

17. The Suggested Upper Merged Ontology. http://ontology.teknowledge.com/

18. The CYC Top-level Ontology. http://www.cyc.com/cyc-2-1/cover.html

19. The SUO Library. http://suo.ieee.org/refs.html

20. The DAML Library. http://www.daml.org/ontologies/ 